\title{
CARACTERIZACIÓN TECNOLÓGICA E ÍNDICES DE CAPTURA DE ELASMOBRANQUIOS, CON REDES DE ENMALLE QUE INCORPORAN ANZUELOS, UTILIZADAS EN EL GOLFO DE SALAMANCA (CARIBE COLOMBIANO)
}

\author{
TECHNOLOGICAL FEATURES AND ELASMOBRANCHS CATCH INDEXES WITH THE \\ HOOK-ADDED GILLNETS USED IN THE SALAMANCA GULF \\ (COLOMBIAN CARIBBEAN)
}

\author{
Cristhian Marrugo, Jairo Altamar y Luis Orlando Duarte
}

\begin{abstract}
RESUMEN
La captura de elasmobranquios asociada a la pesca artesanal en el golfo de Salamanca ha sido poco estudiada. Particularmente, se carece de evaluaciones del impacto que la actividad extractiva produce sobre las abundancias de estos recursos, por lo cual resulta necesario determinar la composición de las capturas de los artes de pesca y las características constructivas y operativas de dichas artes. Mediante análisis de información de desembarcos pesqueros y de conocimiento tradicional, se evaluó la pesquería artesanal de redes de enmalle que opera en el golfo de Salamanca. El análisis evidenció un aumento en el poder de pesca, y la reciente incorporación de anzuelos a las redes de enmalle, cuya práctica inició hace 20 años pero se masificó desde 2010, lo que incrementa la eficiencia en la captura incidental de elasmobranquios. Se documentó una disminución histórica en el tamaño de malla (de 127,0 a 88,9 mm), la cual no introdujo cambios en la tendencia de la captura por unidad de esfuerzo, pero sí estuvo acompañada por una ampliación progresiva de la distribución espacial de la zona de pescas. Según la percepción de los pescadores, la incorporación de anzuelos hace que las redes de enmalle tengan una mayor incidencia en la captura de tiburones. Los resultados obtenidos contribuyen a la identificación de medidas de manejo orientadas a la conservación de elasmobranquios.
\end{abstract}

PALABRAS CLAVE: Artes de pesca, tiburones, elasmobranquios, esfuerzo de pesca, pesca artesanal

\begin{abstract}
Elasmobranchs associated to artisanal fisheries in the Gulf of Salamanca has been studied poorly. In particular, the absence of assessments of the impact that fisheries activity occurs on the abundances of these resources, which is necessary to determine both the composition of the catches of fishing gear that catch elasmobranches as constructive and operational characteristics of these gears. By analyzing information from fishing landings and traditional knowledge, the present study evaluated artisanal gillnet fishery operating in the Gulf of Salamanca. The analysis showed an increase in fishing power, highlighting the recent addition of hooks to gillnets, whose practice began 20 years ago but massified since 2010, increasing efficiency of elasmobranchs catches. A historical decrease in mesh size (from 127.0 to $88.9 \mathrm{~mm}$ ) was documented, which however did not produced changes in the trend of catch per unit of effort, but it was accompanied by a progressive expansion of the spatial distribution of fishing area. As perceived by the fishermen, incorporating hooks makes gillnets have a greater impact on shark catches. The results contribute to the identification of management measures aimed at conservation of elasmobranchs.
\end{abstract}

KEY WORDS: fishing gears, sharks, fishing power, Caribbean Sea, Colombia 


\section{INTRODUCCIÓN}

La sobreexplotación de recursos pesqueros ha sido un tema de interés mundial en los últimos años, se ha detectado la necesidad de incorporar estrategias de manejo para el aprovechamiento sostenible y la conservación de los recursos (Stevens et al., 2000). Las perturbaciones ocasionadas a los ecosistemas marinos por la actividad pesquera, han provocado la disminución en biomasa de grandes vertebrados, con los consecuentes cambios en la estructura de los hábitats y por tanto de la biodiversidad (Lewison et al., 2004; Pinnegar y Engelhard, 2008).Los elasmobranquios son uno de los grupos más vulnerables a la presión pesquera, por sus características de historia de vida y su dependencia de hábitat (Gallager et al., 2012).

Es común la captura incidental de los condrictios en muchas pesquerías, aunque no siempre bien especificados, particularmente aquellas que usan redes de arrastre, palangres o redes de enmalle (Stevens et al., 2000). En el caso de las redes de enmalle se ha documentado que son causantes de altas mortalidades de Mustelus antarcticus y Galeorhinus galeus, en áreas de crianza de Tasmania (Williams y Schaap, 1992) y disminuciones en la abundancia de Carcharias taurus en las costas de la Nueva Gales del Sur (Pollard, 1996). Hay suficiente evidencia en la historia de las pesquerías alrededor del mundo, que sugieren fuertes disminuciones en el tamaño de las poblaciones, tanto por la captura dirigida, como por la pesca acompañante (Stevens et al., 2000; Baum et al., 2003).

Aunque en el golfo de Salamanca (GdeS), Caribe colombiano, se sabe que existe una pesquería artesanal asociada a la captura de elasmobranquios (Yacomelo et al., 2013), con un elevado esfuerzo pesquero (Pardo, 2011), el conocimiento sobre los desembarcos de elasmobranquios es aún limitado, para evaluar el impacto de la pesca sobre estos recursos (Caldas et al., 2009). Las estadísticas históricas no han sido continuas y las existentes presentan una pobre resolución taxonómica en este grupo. La carencia de información sobre estos recursos en el golfo de Salamanca se hace aún más preocupante, si se tiene en cuenta: 1. El acentuado aumento del poder de pesca de la pesquería con redes de enmalle que opera con la incorporación de anzuelos, 2. La marcada tendencia descendente en la densidad de biomasa de algunos recursos pesqueros demersales (García et al., 2007) y 3. El último dato disponible sobre la proporción, entre peces elasmobranquios y peces óseos, que mostró una disminución entre 1970 y 1996 que varió de 0,30 a 0,07, lo que evidencia una reducción relativa de la biomasa en la parte norte de la región Caribe (Vivas-Muñoz et al., 2008).

Los pescadores artesanales modifican de manera continua sus sistemas de extracción, como una estrategia para mejorar el rendimiento de sus capturas. Sin embargo, estos cambios no han sido documentados técnicamente, se encuentran registros en la literatura gris con esquemas poco detallados y no se ha analizado el efecto sobre la captura de elasmobranquios, toda vez que se muestra un aumento en el poder de pesca de las redes de enmalle usadas en esa región. De acuerdo con lo anterior, en el presente trabajo se evalúa el impacto de la incorporación de anzuelos en las redes de enmalle, sobre las abundancias relativas de elasmobranquios, teniendo en cuenta las variaciones espacio-temporales de la captura y los aspectos tecnológicos y operacionales del arte.

\section{MATERIALES Y MÉTODOS}

\section{Área de estudio}

El estudio se llevó a cabo en la población de Puebloviejo (Magdalena), en el sector central del GdeS. Esta zona es considerada de importancia pesquera en el Caribe colombiano (Manjarrés, 2004).

\section{Obtención de la información}

Se realizó una caracterización tecnológica de las redes de enmalle, para lo cual se registraron in situ los parámetros constructivos y operativos del arte. Los parámetros constructivos fueron determinados de acuerdo a la literatura técnica disponible (Fridman y Carrothers, 1986; Okonski y Martini, 1987). Con base en dichos parámetros, se construyó el plano de una red de enmalle típica de la región del GdeS, con la incorporación de anzuelos, siguiendo la normativa de la FAO (Nédélec y Prado, 1990), es decir, se tuvo en cuenta la escala, detalles del armado, materiales de construcción y un esquema de operación de la red.

El análisis de las tasas de captura de elasmobranquios asociadas a las redes de enmalle se basó en dos fuentes informativas: i) el conocimiento ecológico tradicional de los pescadores artesanales, de utilidad demostrada para el estudio de pesquerías con datos limitados (Hall 
y Close, 2007; Cuello y Duarte, 2009), y ii) los registros históricos de desembarcos efectuados en el GdeS entre 1994 y 1998 (Manjarrés et al., 2004), entre 2007 y 2008 (Duarte, 2009) y en 2013 (Duarte, 2014). La eficiencia de captura de las redes que utilizan anzuelos anudados a las boyas se estableció a partir del conocimiento ecológico tradicional de los pescadores que usan este arte de pesca "combinado", a partir de la aplicación de encuestas semi-estructuradas (Bazigos, 1975), como instrumento para la documentación del número de individuos de elasmobranquios capturados semanalmente. Además, se comparó la percepción que tenían los pescadores de las capturas positivas de elasmobranquios realizadas tanto con el anzuelo como con la red.

A partir de la información de desembarcos históricos en el GdeS, se realizó una comparación de la captura por unidad de esfuerzo (CPUE) de años anteriores (de $1994 \mathrm{a}$ 1998, de 2007 a 2008), es decir, cuando no se utilizaban anzuelos en las redes, versus el año más reciente (2014), proveniente del conocimiento ecológico tradicional.

\section{Análisis de la información}

Se calcularon intervalos de confianza de la CPUE (kg día ${ }^{-1}$ ), por de medio de técnicas de bootstrap, con el método de sesgo-corregido y acelerado (Efron, 1987). Para evaluar los cambios en la abundancia relativa se contrastó también el porcentaje de ocurrencia de elasmobranquios en las faenas, lo cual equivale al porcentaje del número de veces en que fueron capturados tiburones y rayas con relación al total de faenas muestreadas con redes de enmalle (porcentaje de capturas positivas).
La distribución espacio-temporal de las capturas de elasmobranquios se analizó mediante mapas temáticos construidos en Qgis versión 2.4 (Quantum GIS, 2014), a partir de la información histórica de desembarcos referenciada espacialmente mediante un sistema de cuadriculas de $1 \mathrm{mn}^{2}$ (Manjarrés et al., 2004).

\section{RESULTADOS}

\section{Caracterización de las redes de enmalle}

La red de enmalle es uno de los artes de pesca artesanal más comunes en el Caribe de Colombia. En el Gdes este arte consta de un paño o la unión de varios paños de poliamida (PA) monofilamento, cuyas dimensiones varían de acuerdo al objetivo de pesca y disponibilidad de los materiales para su construcción. La longitud de las redes de enmalle varían entre 500 y $2100 \mathrm{~m}$, con 40 a 100 mallas de caída, tamaños de ojo de malla entre 50,8 y $88,9 \mathrm{~mm}$, longitud de angola o entralle de $80 \mathrm{a}$ $110 \mathrm{~mm}$ y coeficiente de armado o encabalgadura (E) de 0,65 a 0,78. La relinga superior es de polipropileno (PP), con flotadores que pueden ser de diferentes materiales de reciclaje, es muy común el uso de etil vinil acetato (EVA), los cuales son recortados en forma cilíndrica. La relinga inferior tiene lastres de plomo $(\mathrm{Pb})$ de forma cilíndrica, con un peso que varía entre 100 y $125 \mathrm{~g}$ cada uno. Tanto las distancias entre flotadores como las distancias entre plomos varían entre 1,8 y $2,7 \mathrm{~m}$. Para la señalización y ubicación de la red de enmalle se utilizan recipientes plásticos con capacidad de 4 a 6 galones que hacen las veces de boyas y se utilizan al inicio y al final de cada paño (Figura 1).
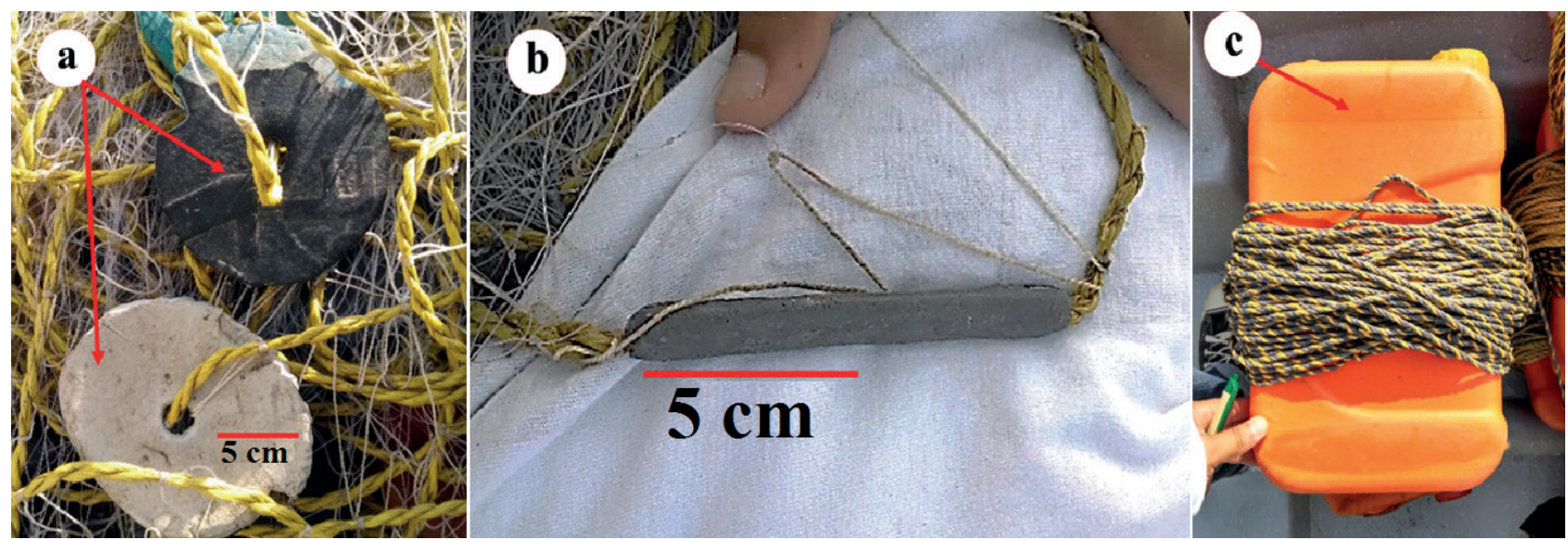

Figura 1. Elementos constructivos de una red de enmalle típica en el Golfo de Salamanca: a) flotadores en material EVA; b) lastre cilíndricos de plomo $(\mathrm{Pb}) ; \mathrm{c})$ recipientes plásticos usados para la señalización de la red de enmalle. 
En estas redes de enmalle es cada vez más frecuente la incorporación de 3 a 10 anzuelos, cada uno de los cuales es anudado individualmente a los recipientes plásticos que actúan como boyas. Se utilizan anzuelos de la marca Mustad, con forma circular o curva, con referencia número 16/0 y el jota o Kirby con referencia número 4 (Figura 2). La longitud de los reinales oscila entre 9 y $68 \mathrm{~m}$, y son construidos de polipropileno (PP) de 5 a $10 \mathrm{~mm}$ de diámetro, en algunos casos reforzados al final con guaya de 1,6 a $2 \mathrm{~mm}$ de diámetro y 1 a 1,5 $\mathrm{m}$ de longitud. El reinal lleva en la parte superior un recipiente de polietileno de alta densidad (PEAD) con capacidad de 1 a $2 \mathrm{l}$, que sirve para ubicar el reinal o detectar una posible captura debido a su hundimiento, además de evitar que el anzuelo se enrede con la red de enmalle. En la Figura 3, se muestra el plano de una red de enmalle típica de la región del GdeS, con la ubicación y características de los anzuelos incorporados.

Las redes de enmalle operan fijas en el fondo por un periodo de 5 a 6 horas, son revisadas a intervalos de 1 a 2 horas, para extraer las capturas tanto de las redes como de los anzuelos. En cada faena de pesca intervienen de 3 a 5 pescadores, este número depende de la capacidad de las embarcaciones, las cuales tienen casco de fibra de vidrio o de madera recubierta con fibra de vidrio, esloras de 5 a $8 \mathrm{~m}$, mangas de 1 a $2 \mathrm{~m}$ y motores fuera de borda cuya potencia varía entre 15 y 75 HP. Los sitios de pesca son caladeros de pesca o lugares conocidos por los pescadores, que pueden cambiar de acuerdo al clima y la especie objetivo.

\section{Percepción de los pescadores}

De acuerdo con los resultados obtenidos de la percepción de 67 pescadores encuestados, el $65 \%$ expresó haber incorporado anzuelos en las redes de enmalle cinco años atrás, mientras que solo tres pescadores afirmaron haber utilizado anzuelos en las redes hace 20 años. Además, se detectó según esta fuente que las redes capturan de 1 a 6 elasmobranquios por semana (la respuesta más frecuente fue de 1 a 2 elasmobranquios por semana). Los pescadores también informaron que la mayoría de los individuos son capturados por los anzuelos y no por las redes (Figura 4).

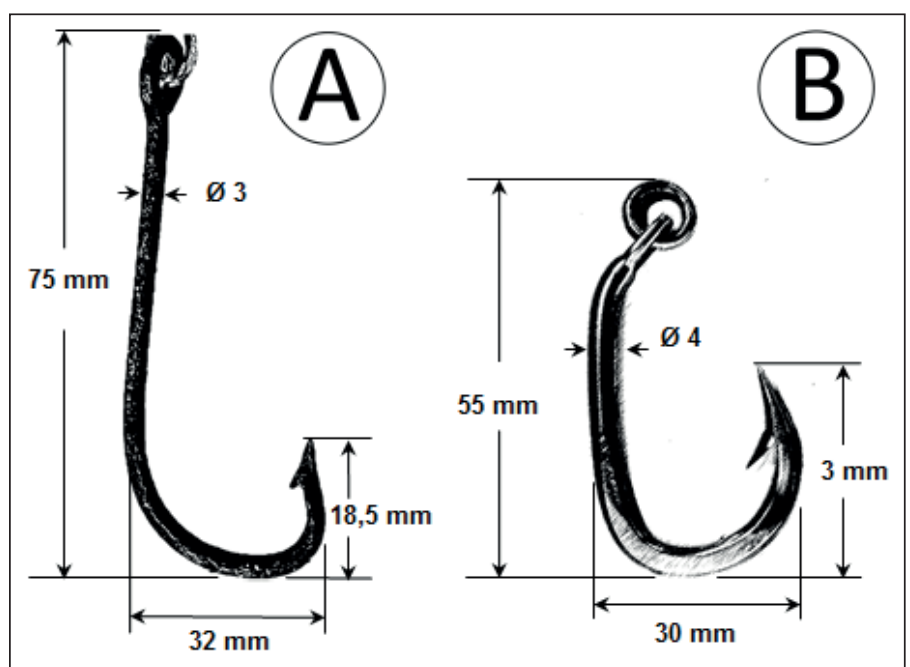

Figura 2. Tipos de anzuelos que se incorporan a las redes de enmalle para la captura de elasmobranquios: (a) anzuelo Kirby o tipo J, (b) anzuelo curvo.

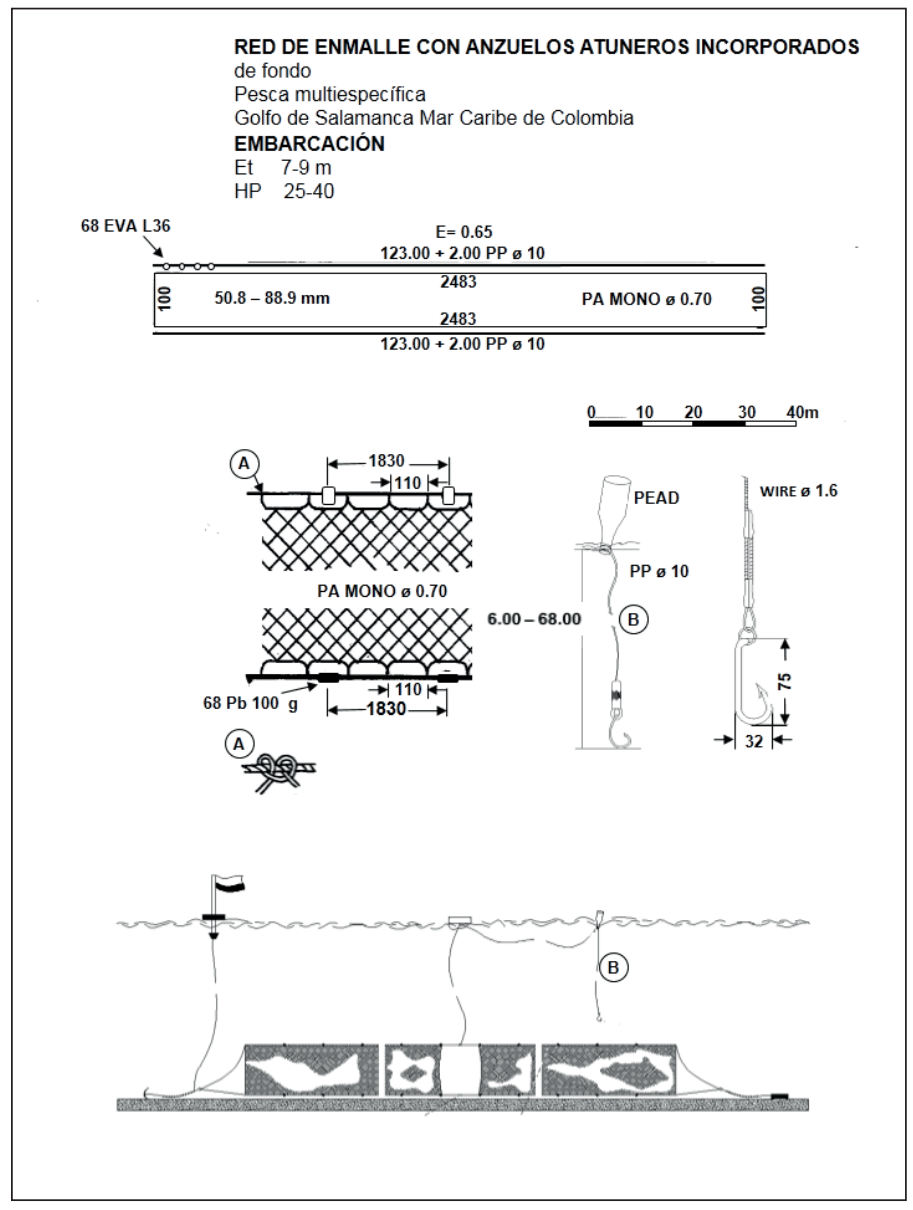

Figura 3. Plano de una red de enmalle comúnmente utilizada en la pesca artesanal del Golfo de Salamanca, mostrando los detalles de la incorporación de anzuelos. 


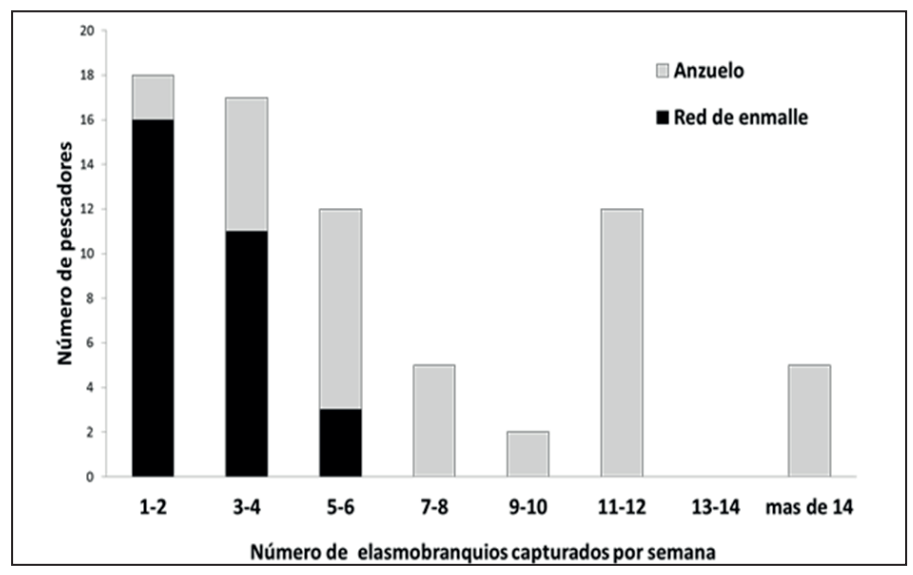

Figura 4. Frecuencias de captura de elasmobranquios en las redes de enmalle según la percepción de los pescadores artesanales.

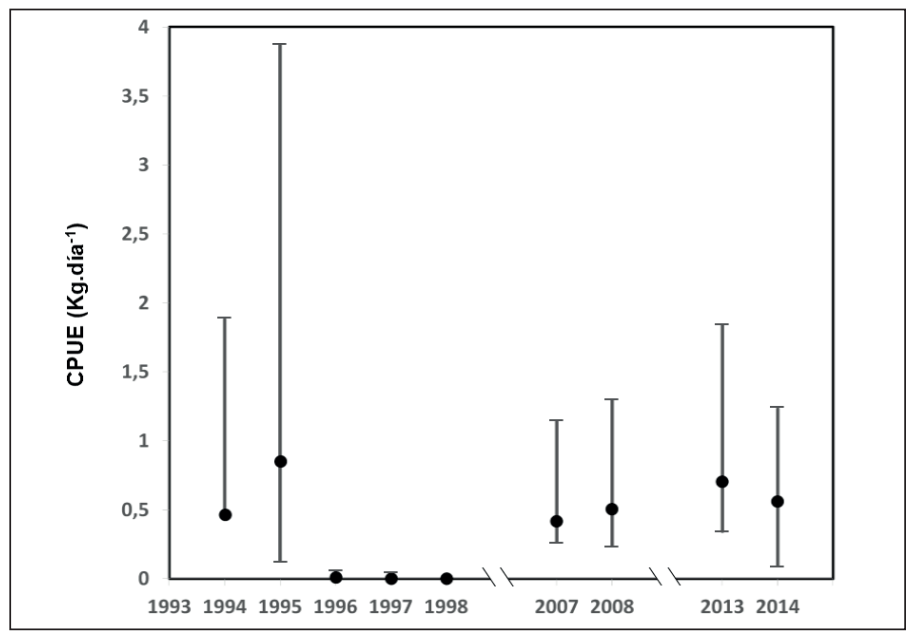

Figura 5. Variación interanual de la CPUE de elasmobranquios de la pesquería artesanal de redes de enmalle en el Golfo de Salamanca.

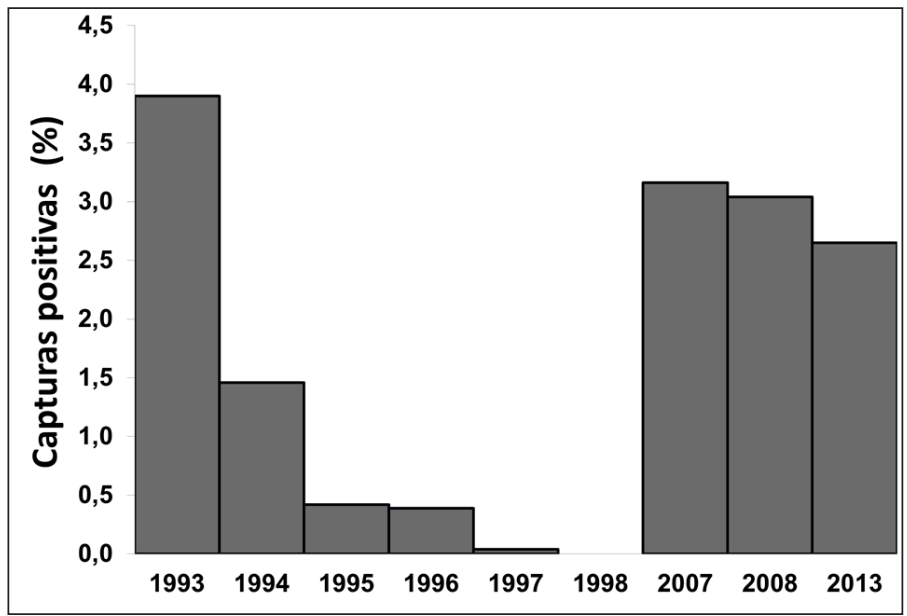

Figura 6. Porcentaje de capturas positivas para elasmobranquios en las faenas de la pesquería de redes de enmalle del Golfo de Salamanca muestreadas durante los períodos 1994-2008 y 2013.
Variaciones espacio-temporales de las capturas de elasmobranquios

En lo que respecta a las abundancias relativas, la comparación de la CPUE promedio de elasmobranquios en los diferentes periodos mostró una alta variabilidad al inicio de la serie, pero en general no se evidencia tendencia alguna. Cabe destacar, la notable disminución en la CPUE de elasmobranquios durante el período 1996-1998 (Figura 5).

La pobre resolución taxonómica no permitió realizar una estimación de la abundancia relativa (CPUE) por especie. Por tanto, se estimó el porcentaje de capturas positivas (presencia) de elasmobranquios en los periodos 1994-1998 y 2007-2008. Este análisis mostró una disminución de las capturas positivas de elasmobranquios entre 1993 y 1998, mientras que aumentaron significativamente en el período 2007-2008 y en 2013 (Figura 6).

Se encontró que las capturas de elasmobranquios con redes de enmalle han experimentado cambios históricos en su distribución espacial. En el periodo 1993-1998, se evidenció una distribución muy restringida de los sitios de capturas positivas para elasmobranquios, los cuales se concentraron básicamente en dos sectores: la parte nororiental del GdeS y la parte central del GdeS. A diferencia de lo anterior, durante el año 2014 la distribución de estos sitios abarcó también la parte occidental del Gdes, hacía la desembocadura del rio Magdalena, señal de que los pescadores han ampliado la cobertura espacial de sus zonas de pesca (Figura 7).

\section{DISCUSIÓN}

En el Caribe colombiano la pesca artesanal se ha caracterizado por la implementación de diferentes métodos de pesca, teniendo especialidades o métodos según el objetivo de captura (Escobar et al., 2013). En el GdeS las redes de enmalle son el arte de pesca más común y se han evidenciado cambios históricos en sus características constructivas y operativas. Los principales cambios han sido la disminución del tamaño de malla y del coeficiente de armado (Tabla 1). El tamaño de 
malla modal ha disminuido de 127,0 a $88,9 \mathrm{~mm}$, por tanto, es de esperarse que se incremente la captura de individuos más pequeños (Erzini et al., 2003), ya que la selectividad de las redes de enmalle disminuye. De acuerdo a la disponibilidad de los recursos pesqueros y a las características de operación, suelen observarse cambios en los aspectos constructivos que influyen en la selectividad y en el poder de pesca (Pope et al., 1983; Cubillos et al., 1998; Machiels et al., 1994).

Otra de las estrategias utilizadas por los pescadores para mejorar los rendimientos pesqueros es la reducción del coeficiente de armado de la red, que permite aumentar la captura por enredamiento haciéndolas menos selectivas. Pardo (2011), documentó una disminución apreciable de este coeficiente, sin embargo, en el caso de algunos tiburones, se ha demostrado que el efecto del coeficiente de armado y el de algunas dimensiones de anzuelos sobre las tasas de capturas era débil. Un hecho reciente que evidencia la permanente búsqueda de mayores tasas de captura por parte de los pescadores, es la incorporación de anzuelos en las redes de enmalle, orientados particularmente a la captura de elasmobranquios, lo cual confirma la existencia de una pesca asociada a estas poblaciones en el GdeS (Yacomelo et al., 2013).

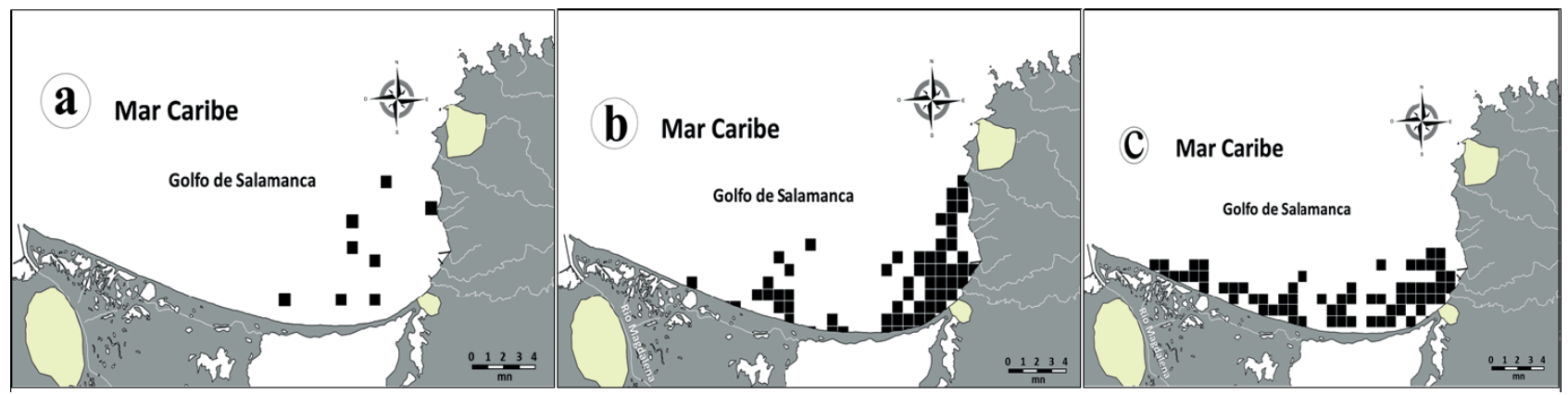

Figura 7. Variación temporal de la distribución espacial de los sitios de capturas positivas para elasmobranquios. a) período 1993-1998, b) período 2007-2008 y c) 2014.

Tabla 1. Cambios históricos en los aspectos constructivos en las redes de enmalle que operan en el golfo de Salamanca.

\begin{tabular}{ccccc}
\hline Material del paño & Tipo & Tamaño de malla (mm) & Coeficiente de armado (E) & Referencias \\
\hline Poliamida (PA) & & $118,0-127,0$ & 0,50 & Hernández, A. (1986) \\
Poliamida (PA) & Monofilamento & $88,9-101,0$ & 0,79 & Manjarrés et al.(1993) \\
Poliamida (PA) & Monofilamento & $76,2-114,3$ & - & Barros y Manjarrés (2004) \\
Poliamida (PA) & Monofilamento & $50,8-76,0$ & 0,40 & Pardo (2011) \\
Poliamida (PA) & Monofilamento & $69,9-76,2$ & $0,61-0,82$ & Salcedo (2014) \\
Poliamida (PA) & Monofilamento & $50,8-88,9$ & $0,65-0,78$ & Presente estudio \\
\hline
\end{tabular}

En el área norte del Caribe colombiano, la pesquería artesanal de enmalle captura un amplio número de especies, como Euthynnus alletteratus, Caranx crysos, Caranx hippos, Conodon nobilis, Lutjanus analis, Micropogonias furnieri, Scomberomorus brasiliensis, Scomberomorus cavalla, Trichiurus lepturus, cuyos porcentajes de inmaduros capturados sobrepasan el 50\% (Altamar et al., 2015), lo cual conlleva a la sobrepesca de crecimiento y la disminución en la abundancia de las poblaciones explotadas (Carlson y Cortés, 2003; Walker, 1998). Esta problemática de disminución del tamaño de malla, quizás está relacionada al hecho de que los pescadores artesanales habitualmente modifican sus sistemas de extracción, como una estrategia para mejorar o mantener el rendimiento de las capturas. 
En el caso de las tasas de captura de elasmobranquios en el GdeS, no se encontró una tendencia en particular durante el periodo de estudio, aunque se observó un aumento en el porcentaje de faenas que capturaron elasmobranquios. Se ha planteado que las capturas de elasmobranquios en el área están asociadas a las épocas del año de mayor disponibilidad de nutrientes (Manjarrés, 2004; Párraga et al., 2010), que resultan con poca variación en la escala temporal corta que se analizó. No obstante, a más largo plazo (1970 a 2001), existe evidencia de una disminución histórica en los elasmobranquios demersales en la zona norte del Caribe continental de Colombia (García et al., 2007), situación también observada en los elasmobranquios del Atlántico noroccidental y del mar Caribe (Baum y Myers, 2004; Baum et al, 2003).

Una dimensión que debe considerarse para interpretar las tendencias de variación en las capturas es la espacial. Se observó que los pescadores han venido ampliando sus zonas de pesca a lo largo del período analizado. Es posible que la disminución local de las abundancias de elasmobranquios y el incremento en la autonomía de las embarcaciones, sean factores que han promovido esa ampliación de las zonas de pesca. Si bien, debe tenerse en cuenta que la distribución espacial del esfuerzo de pesca en el GdeS, varía según la época climática (Pardo, 2011), probablemente por limitaciones en la navegabilidad de las embarcaciones que allí operan. Detectar estas variaciones espacio-temporales permite tener una mejor perspectiva para la toma de decisiones en el marco de un buen manejo pesquero (Munro, 1973; Manjarrés, 2010).

El estudio determinó la existencia de una pesquería artesanal de redes de enmalle que a través de modificaciones se ha orientado a la captura incidental de tiburones y rayas en el GdeS, lo cual fue comprobado mediante el aumento en la frecuencia relativa de desembarcos con capturas positivas de tiburones y rayas y a través de la información derivada del conocimiento tradicional de pescadores artesanales de la región. Las evidencias indican que esta pesquería ha incrementado el poder de pesca sobre las poblaciones de elasmobranquios y por tanto se requieren evaluar posibles impactos sobre las poblaciones capturadas 90 con el fin de establecer medidas de manejo pesquero apropiadas.

\section{AGRADECIMIENTOS}

Estudio auspiciado por Colciencias (Cod.1117-52129352) y la Universidad del Magdalena. La AUNAP auspició parcialmente la investigación (Convenio 11 de 2012). Los autores expresan su agradecimiento a los pescadores artesanales del Golfo de Salamanca por permitir el registro de su actividad.

\section{REFERENCIAS}

Altamar, J., Manjarrés-Martinez, L., Duarte, L.O., Cuello, F. y Escobar - Toledo, F. 2015. ¿Qué tamaños deberíamos pescar? Autoridad Nacional de Acuacultura y Pesca (AUNAP) - Universidad del Magdalena, Santa Marta.

Barros, M. y Manjarrés-Martinez, L. 2004. Inventario y caracterización general de la flota pesquera artesanal del sector norte del departamento del Magdalena (Taganga - La Jorara). En: Manjarrés-Matinez, L. Pesquerías demersales del área norte del Mar Caribe de Colombia y parámetros biológico-pesqueros y poblacionales del recurso pargo. Universidad del Magdalena, Santa Marta.

Baum, J.K. y Myers, R.A. 2004. Shifting baselines and the decline of pelagic sharks in the Gulf of Mexico. Ecology Letters 7(2): 135-145.

Baum, J.K., Myers, R.A., Kehler, D.G., Worm, B., Harley, S.J., y Doherty, P.A. 2003. Collapse and conservation of shark populations in the Northwest Atlantic. Science 299(5605): 389-392.

Bazigos, G.P. 1975. Esquema de encuestas sobre estadística de pesca: Aguas continentales. Organización de las Naciones Unidas para la Agricultura y la Alimentación, FAO.

Caldas, J.P., Castro-González, E., Puentes, V., Rueda, M., Lasso, C., Duarte, L.O., Grijalba-Bendeck, M., Gómez, F., Navia, A.F., Mejía-Falla, P.A., Bessudo, S., Diazgranados, M.C. y Zapata Padilla, L.A. 2009. Estado del conocimiento de los peces cartilaginosos del Caribe continental de Colombia. En: Puentes, V., Navia, A.F., Mejía-Falla, P.A., Caldas, J.P., Diazgranados, M.C. y Zapata, L.A. Avances en el conocimiento de tiburones, rayas y quimeras de Colombia. Fundación Squalus, Ministerio de Ambiente, Vivienda y Desarrollo Territorial, Instituto Colombiano Agropecuario, Colciencias, Conservación internacional, WWF. 
Carlson, J.K. y Cortés, E. 2003. Gillnet selectivity of small coastal sharks off the southeastern United States. Fisheries Research 60: 405 -414

Cubillos, L., Canales, M., Hernández, A., Bucarey D., Vilugrón, L. y Miranda, L. 1998. Poder de pesca, esfuerzo de pesca y cambios estacionales e interanuales en la abundancia relativa de Strangomera bentincki y Engraulis ringen en el área frente a Talcahuano, Chile (1990-1997). Investigaciones Marinas, Valparaíso 26: 3-14

Cuello, F. y Duarte, L.O. 2009. El Pescador Artesanal, Fuente de Información Ecológica para la Ordenación Pesquera en el Mar Caribe de Colombia. Proceedings Gulf Caribbean Fisheries Institute 62: 454-470.

Duarte, L.O. (Ed.) 2009. Construcción participativa de una propuesta integral para la conservación de los recursos hidrobiológicos en dos áreas protegidas del Caribe de Colombia y para su uso sostenible en las zonas adyacentes. Informe Técnico, Colciencias, Universidad del Magdalena. Santa Marta.

Duarte, L.O. (Ed.) 2014. Condrictios: evaluación de la pesca de Condrictios en el Caribe de Colombia: atributos bioecológicos, socioeconómicos y pesqueros para la conservación y manejo del recurso en la región. Informe Técnico, Colciencias, Universidad del Magdalena. Santa Marta.

Efron B. 1987. Better bootstrap confidence intervals. Journal of the American Statistical Association 82: 171-185.

Erzini, K., Gonçalves, J., Bentes, L., Lino, P.G., Ribeiro, J. y Stergiou, K.I. 2003. Quantifying the roles of competing static gears: comparative selectivity of longlines and monofilament gill nets in a multi-species fishery of the Algarve (southern Portugal). Scientia Marina 67(3): 341-352.

Escobar, F., Parrado-Cortes M., Duarte, L.O. y ZetinaRejon, M. 2013. Incidencia de la Pesca Artesanal Sobre la Diversidad Taxonómica y Funcional de la Comunidad de Peces en el Mar Caribe de Colombia. Proceedings Gulf Caribbean Fisheries Institute 62: 346 - 351.

FAO. 1987. Catálogo de artes de pesca artesanal. Segunda edición. Fishing News Books Ltd. Farnham, Surrey.

Fridman, A. y Carrothers, P. 1986. Calculations for fishing gear designs. Fishing Manuals Food and Agriculture Organization, FAO.

Gallager, A.J., Kine, P.M. y Hammerschlag, N. 2012. Ecological risk assessment and its application to elasmobranch conservation and management. Journal of Fish Biology 80(5): 1727-1748.
García, C.B., Duarte, L.O., Altamar, J. y Manjarrés, L. 2007. Demersal fish density in the upwelling ecosystem off Colombia, Caribbean Sea: Historic outlook. Fisheries Research 85(1-2): 68-73.

Hall, G.B. y Close, C.H. 2007. Local knowledge assessment for a small-scale fishery using geographic information systems. Fisheries Research 83(1): 11-22.

Hernández, A. 1986. Desarrollo de la pesca artesanal en la región de Santa Marta. COLCIENCIAS-CIID-FES, Ed. Guadalupe, Bogotá.

Lewison, R.L., Crowder, L.B., Read, A.J., y Freeman, S.A. 2004. Understanding impacts of fisheries bycatch on marine megafauna. Trends in Ecology y Evolution 19(11): 598-604.

Machiels, M.A.M., Klinge, M., Lanters, R. y Van Densen, W.L.T. 1994. Effect of snood length and hanging ratio on efficiency and selectivity of bottom-set gillnets for pikeperch, Stizostedion lucioperca L., and bream Abramis brama. Fisheries Research 19 (3-4): 231-239.

Manjarrés, L.M. 2004. Pesquerías demersales del área norte del Mar Caribe de Colombia y parámetros biológicopesqueros y poblacionales del recurso pargo. Universidad del Magdalena, Santa Marta.

Manjarrés, L.M., Infante-Rueda, J. y Escorcia, F. 1993. Evaluación de captura y esfuerzo pesquero en el área marítima de Santa Marta. Proyecto integral de investigaciones y desarrollo de la pesca artesanal marítima en el área de Santa Marta. Informe Técnico final.

Manjarrés, L.M., Mazenet, J.C., Duarte, L.O., Infante, J.H. y Cuello, F. 2004. Guía de usuario de las bases de datos pesqueros artesanales de los departamentos del Magdalena y La Guajira, pp. 12-35. En: Manjarrés, L.M. (ed.) Estadísticas pesqueras artesanales del Magdalena y La Guajira, con aplicación de herramientas informáticas para su sistematización y procesamiento. Universidad del Magdalena, COLCIENCIAS, INPA, INCODER. Santa Marta, $71 \mathrm{p}$

Manjarrés-Matinez, L. Gutiérrez, J.C., Mazenet J. y Soriguer, M.C. 2010. Seasonal patterns of three fish species in a Caribbean coastal gill-net fishery: Biologically induced or climate-related aggregations? Fisheries Research 106 (3):358-367.

Munro, J.L., Guat, V.C., Thompson, R. y Reeson, P.H. 1973. The spawning seasons of the Caribbean reef fishes. Journal Fisheries Biology 5: 69-84.

Nédélec, C. y Prado, J. 1990. Definition and classification of fishing gear categories. Fishing Manuals: Fisheries Technical, Food and Agriculture Organization, FAO. 
Okonski, S.L. y Martini, L.W. 1987. Artes y Métodos de pesca. Materiales didácticos para la capacitación técnica. Hemisferio Sur.

Pardo, E. 2011. Efectos de las artes de pesca sobre la estructura de tallas de pargo rayado (Lutjanus synagris) en el Golfo de Salamanca, mar Caribe de Colombia. Tesis de Grado, Universidad del Magdalena. Santa Marta, Magdalena, Colombia.

Párraga, D.P., Cubillos, L.A. y Correa, M.A. 2010. Variaciones espacio-temporales de la captura por unidad de esfuerzo en la pesquería artesanal costera del pargo rayado Lutjanus synagris, en el Caribe colombiano y su relación con variables ambientales. Revista de Biología Marina y Oceanografía 45 (1): 77-88.

Pinnegar, J.K., y Engelhard, G.H. 2008. The 'shifting baseline' phenomenon: a global perspective. Reviews in Fish Biology and Fisheries 18(1): 1-16.

Pollard, D.A. 1996. The biology and conservation status of the grey nurse shark (Carcharias taurus Rafinesque 1810) in New South Wales, Australia. Aquatic Conservation: Marine and Freshwater Ecosystems 6: 1-20.

Pope, J.A., Margetts, A.R., Hamley, J. y Akyuz, E.F. 1983. Manual de métodos para la evaluación de las poblaciones de peces. Parte 3. Selectividad de arte de pesca. Documento Técnico de Pesca Roma, Organización de las Naciones Unidas para la Agricultura y la Alimentación FAO.

Quantum GIS (QGIS) Versión 2.4. 2014. QGIS Development Team. http// www.qgis.org/.
Salcedo, J. 2014. Caracterización de la pesquería artesanal asociada a los desembarcos de tiburones y rayas en el Golfo de Salamanca-Colombia. Tesis de Grado, Universidad del Magdalena. Santa Marta, Magdalena, Colombia.

Steven, J., Bonfil, R., Dulvy, N. y Walker, P. 2000. The effects of fishing on sharks, rays and chimaeras (Chondrichthyans), and the implications for marine ecosystems. ICES Journal of Marine Science. 57: 476-479.

Vivas-Muñoz, J., Duarte, L.O. y Garcia, C. 2008. Exploración de tendencias históricas de indicadores trofodinámicos en los peces demersales del mar Caribe de Colombia. Proceedings of the Gulf And Caribbean Fisheries Institute 60: 338 - 344.

Williams, H. y Schaap, A. H. 1992. Preliminary results of a study into the incidental mortality of sharks in gill-nets in two Tasmanian shark nursery areas. Marine and Freshwater Research, 43(1): 237-250.

Yacomelo, J., González, A.F. y Duarte, L.O. 2013. Composición y distribución espacial de las capturas de elasmobranquios en la pesquería artesanal del Golfo de Salamanca, mar Caribe de Colombia, Proceedings Gulf Caribbean Fisheries Institute 65: 54.

Walker, T.I. 1998. Can shark resources be harvested sustainably? A question revisited with a review of shark fisheries. Marine and Freshwater Research 49(7): 553-572.

Fecha de recepción: 06/10/2015 Fecha de aceptación: 13/11/2015

Para citar este artículo: Marrugo, C., J. Altamar y L.O. Duarte. 2015. Caracterización tecnológica e índices de captura de elasmobranquios con redes de enmalle que incorporan anzuelos, utilizadas en el golfo de Salamanca (Caribe colombiano). Revista Intropica Vol. 10: 84 - 92 